\title{
Comparison of the skin microbiota of patients with acne vulgaris and healthy controls
}

\author{
Jian Shi, Jia-Wei Cheng, Qi Zhang, Zhi-Xiang Hua, Xu Miao \\ Department of Dermatology, The Second Affiliated Hospital of Nantong University, Nantong, China \\ Contributions: (I) Conception and design: J Shi; (II) Administrative support: X Miao; (III) Provision of study materials or patients: JW Cheng; (IV) \\ Collection and assembly of data: Q Zhang; (V) Data analysis and interpretation: J Shi; (VI) Manuscript writing: All authors; (VII) Final approval of \\ manuscript: All authors. \\ Correspondence to: Xu Miao. Department of Dermatology, The Second Affiliated Hospital of Nantong University, No. 6 Haierxiang North Road, \\ Chongchuan District, Nantong 226001, China. Email: jsntmcsj@163.com or mx730129@163.com.
}

\begin{abstract}
Background Acne vulgaris is a chronic inflammatory skin disease of the pilosebaceous units which can affect the individual's physiological and psychological health. Abnormal growth of lipophilic anaerobic bacteria such as Propionibacterium acnes is reported to be a major factor in the development of acne. However, the relationship between skin microorganisms and acne has not been fully elucidated. Our study aimed to explore the microbial differences between patients with acne and healthy controls (HCs).

Methods: The study involved 16 participants diagnosed with acne vulgaris and 5 HCs. We collected skin microbe samples from the cheeks, brow, forehead, neck, chin, or chest of the participants with sterile cotton swabs depending on the location of the acne lesions. Cutaneous microbe samples from the participants were tested by 16 s sequencing.

Results: Patients with acne showed increased diversity of skin microbiota in their samples. OTU535601 (Lachnospiraceae), OTU4460604 (Clostridiales), OTU3217705 (Moraxellaceae), OTU1066814 (Prevotella), and OTU455671 (Lactococcus garvieae) were the top 5 most abundant species found in patients with acne but were not present in HCs. OTU423327 (Achromobacter), OTU4423360 (Stenotrophomonas), OTU993127 (Porphyromonas), OTU677680 (Prevotella), and OTU269901 (Pseudomonas) were the top 5 most abundant species in HCs but were not found in patients with acne.

Conclusions: The present study has analyzed and compared the diversity and abundance of microorganisms and the characteristics of the main pathogenic bacteria in patients with acne and HCs. Our findings indicate the importance of maintaining the skin's commensal microflora balance with the development of acne vulgaris.
\end{abstract}

Keywords: Acne vulgaris; skin microbiota; bacterial diversity

Submitted May 13, 2021. Accepted for publication Jun 29, 2021.

doi: 10.21037/apm-21-1482

View this article at: https://dx.doi.org/10.21037/apm-21-1482

\section{Introduction}

The skin, like the gut and other body tissues, is colonized by a dense symbiotic microbial community, which form numerous communities and occupy different cutaneous environmental niches (1). Skin microorganisms have essential roles in protecting against invading pathogens, educating the immune system and breaking down natural products (2). They vary depending on the individual, body site, age, and disease status (3). Many common skin diseases, including acne vulgaris, are related to changes in the microbiota, known as dysbiosis (4), which are often driven by common commensal species (2).

Acne vulgaris (commonly called acne) is a chronic recurrent inflammatory skin disease that typically starts in early puberty. It entails increased facial sebum production resulting in the formation of mid-facial comedones and 
subsequently, inflammatory lesions (5). Broadly, acne affects $85 \%$ of adolescents and young adults, and approximately $20 \%$ of cases are reported to be moderate or severe (6). There are 4 main factors that contribute to the pathogenesis of acne: increased sebum production, inflammatory mediators in the skin, alterations in the keratinization process, and follicular colonization by Propionibacterium acnes ( $P$. acnes).

Although the association between $P$. acnes and acne vulgaris has been confirmed, few studies have investigated the entire facial skin microbiota of acne patients. Hence, the relationship between skin microorganisms and acne has yet to be fully elucidated. Different from some previous reports $(7,8)$, our research focuses on the population in east of China. The present study aimed to detect the differences in the bacterial microbiota of patients with acne and healthy controls (HCs). We present the following article in accordance with the STROBE reporting checklist (available at https://dx.doi.org/10.21037/apm-21-1482).

\section{Methods}

\section{Study participants}

This study included 16 patients diagnosed with acne vulgaris (11 males and 5 females) and 5 HCs (3 males and 2 females). All participants were recruited from the Second Affiliated Hospital of Nantong University. Mild, moderate, and severe acne were defined as S1, S2, and $\mathrm{S} 3$, respectively, in line with the criteria of the Japanese Acne Study Group (9). The participants ranged in age from 16 to 30 years old. All participants included in this study were treatment-naïve, having never received any oral medicine for their condition (e.g., antibiotics, isotretinoin, herbs). Written informed consent was obtained from each participant before enrolment. All procedures performed in this study involving human participants were in accordance with the Declaration of Helsinki. The study was approved by the ethics committee of the Second Affiliated Hospital of Nantong University (No: 2019YKS059) and informed consent was taken from all the patients.

\section{Microbiota sampling}

Participants avoided cleansing the skin, deodorants, makeup, and applying ointment within 24 hours before sampling. And the operator wore sterile gloves during performing the sampling process. Depending on the location of the acne lesions, skin microbiota samples were collected from the cheeks, brow, forehead, neck, chin, or chest of the participants with sterile cotton swabs. The sampling site was scrubbed 20 times with the cotton swab: 10 times in 1 direction and 10 times perpendicular to the first direction. The swabs were placed on a phosphate buffered saline solution and stored immediately at $-20^{\circ} \mathrm{C}$ ahead of DNA extraction.

\section{DNA extraction, PCR amplification, and sequencing.}

DNA was extracted from the samples using the DNeasy PowerSoil Kit (QIAGEN, \# 12888-100) following the manufacturer's instructions. The $\mathrm{V} 3-\mathrm{V} 4$ region of the $16 \mathrm{~S}$ ribosomal RNA (rRNA) gene was amplified by PCR using forward primer 343F and reverse primer 798R (10). After purification and recovery, PCR products were barcoded and quantified with a QuantiFluor-ST Fluorometer (Promega, USA) and then mixed for sequencing. Sequencing was obtained on the Illumina ${ }^{\circledR M i S e q}$ sequencer (Illumina, San Diego, CA, USA) by OE Biotech Co, Ltd. (Shanghai, China).

\section{Sequence data analysis}

QIIME 1.9.1 was used to process the resulting sequence data (11). Briefly, high quality (quality value >25) sequence data derived from the sequencing process were demultiplexed. Operational taxonomic units (OTUs) with 97\% sequence similarity were obtained with USEARCH. All representative reads were annotated and blasted against Greengens using the RDP Classifier. Principle component analysis (PCA) was conducted to assess the variation between the experimental groups. The alpha diversity of all samples was calculated. Analysis of similarities (ANOSIM) and Pearson's correlation analysis were conducted with R.

\section{Statistical analysis}

Data were expressed as mean \pm standard deviation (SD) (12). Differences in the OTUs of patients with acne and HCs were used by the Wilcoxon test $(\mathrm{P}<0.05)$. Probability values $<0.05$ were taken to indicate statistical significance. All analyses were performed using GraphPad Prism version 7.0(GraphPad Software Co, USA). Statistically significant differences $(\mathrm{P}<0.05)$ in the component of skin microbial community between patients 
with acne and HCs were determined using the nonparametric statistical test ANOSIM.

\section{Results}

\section{Background characteristics of study cobort}

This study included 16 patients with acne and $5 \mathrm{HCs}$, whose background characteristics are shown in Table 1. The study participants ranged in age from 16 to 30 years old; the average age was 22.5 and 22.4 years old in the acne and HC groups, respectively. In the patient group, there were 4,8 , and 4 cases of mild, moderate, and severe acne, respectively. Sample collection sites included the cheek $(n=7)$, forehead $(\mathrm{n}=9)$, brow $(\mathrm{n}=1)$, neck $(\mathrm{n}=2)$, chest $(\mathrm{n}=1)$, and $\operatorname{chin}(\mathrm{n}=1)$ (Table S1). All skin samples met the standard for analysis. No participants withdrew from the study, and there were no missing data.

\section{Patients with acne have increased gut microbiota diversity}

Sequencing of $16 \mathrm{~S}$ rRNA genes from variable regions

Table 1 Background characteristics of patients with acne and healthy controls

\begin{tabular}{lccc}
\hline Factors & Normal & Acne & P value \\
\hline Sex $(\mathrm{F} / \mathrm{M})$ & $5(2 / 3)$ & $16(5 / 11)$ & \\
Age, years, mean \pm SD & $22.4 \pm 5.55$ & $22.50 \pm 4.34$ & 0.97 \\
\hline
\end{tabular}

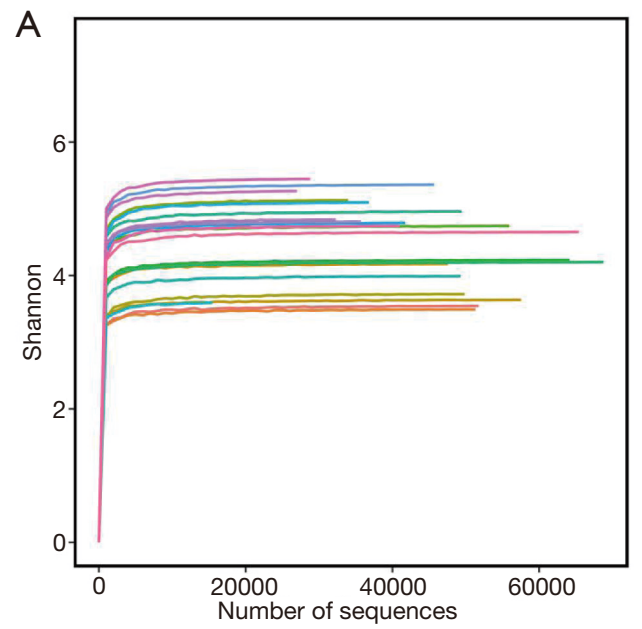

V3-V4 of the skin samples was performed using Illumina MiSeq platforms. A total of 1,372,031 clean reads were obtained, with an average of $65,335 \pm 3,769$ reads per sample after removing unqualified sequences. Shannon index analysis showed that the depth of sequencing covered rare new phylotypes and most microbial diversity (Figure 1A). Compared with the HC group, the acne group exhibited significantly increased microbial diversity, as calculated by the Shannon diversity index $(\mathrm{P}<0.001$, Figure $1 B)$.

\section{Bacterial taxa differences between patients with acne and HCs}

PCA analysis was employed to obtain a comprehensive insight into the microbiota of patients with acne. The results of the acne and HC groups were mainly distributed in 2 clusters (Figure 2A). The clustering of the microbiota composition of the acne group was different from that of the HC group, suggesting distinct community constitutions between two groups. ARISA-based ANOSIM of the 2 groups further confirmed that the intergroup disparities were more remarkable $(\mathrm{R}=0.8123, \mathrm{P}<0.001)$ than intragroup disparities, indicating a significant difference in the skin microbiota of the acne group and HC group. Other factors were also included in the PCA analysis, including sampling sites (Figure 2B), sex (Figure 2C), and acne severity (Figure 2D); however, a lack of distinct clustering between groups and only a small degree of difference in the ANOSIM results (Table S2) indicated that these factors

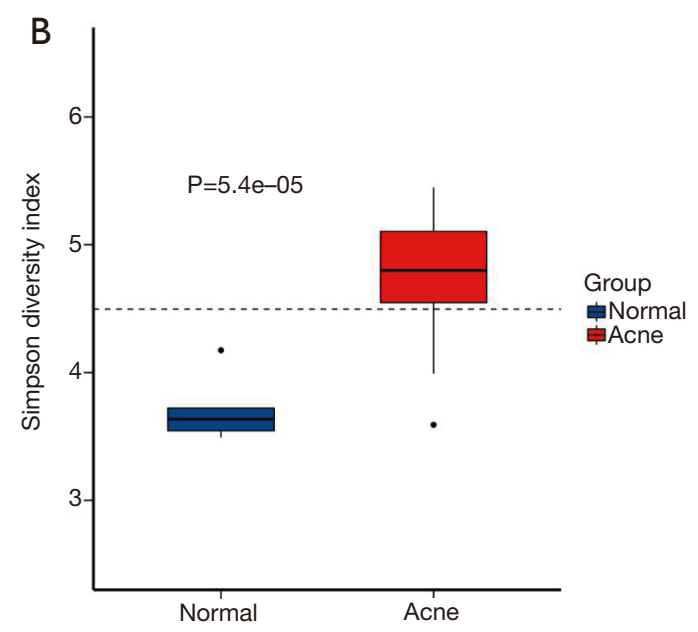

Figure 1 Shannon diversity indexes of acne and HC samples. (A) Shannon diversity curve. (B) Box plot of Simpson diversity index between the acne and HC groups. HC, healthy control. 

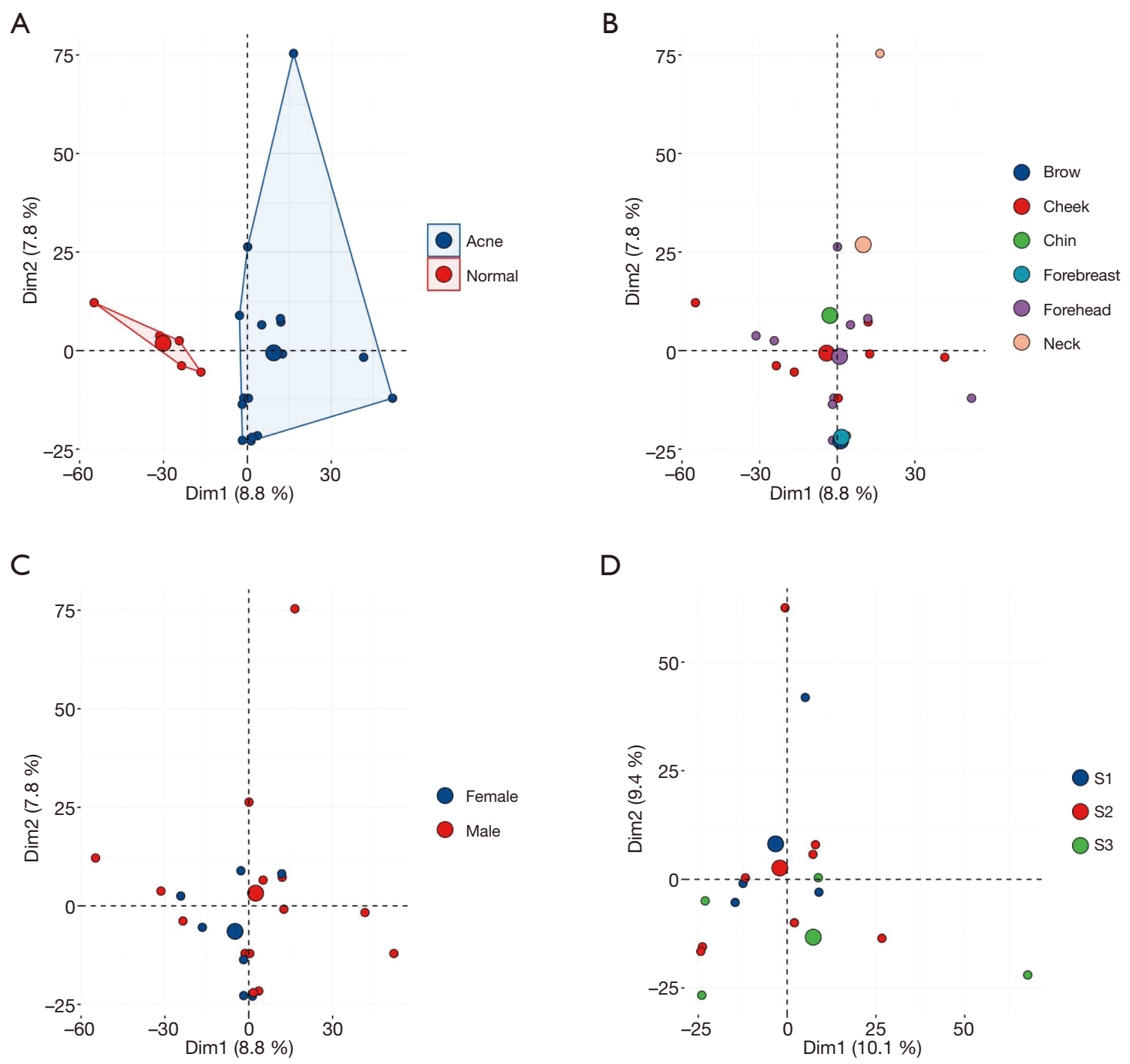

Figure 2 PCA analysis of microbiota between samples. (A) PCA plot between the acne and HC samples. (B) PCA plot between body parts. (C) PCA plot between female and male participants. (D) PCA plot of acne samples classified by disease severity. PCA, principal component analysis; HC, healthy control.

did not have a major influence on the skin microbiota composition of participants in this study.

To further investigate the differences in bacterial composition between the groups, the bacterial taxa in the phylum and genus levels of samples were plotted (Figure 3). Compared with the HC group, the abundance of Firmicutes was increased and that of Proteobacteria was reduced in the acne group. Furthermore, at the genus level, Acinetobacter showed a sharp reduction and many other species showed considerable turbulence in the acne group.

\section{OTU-level analysis of the skin microbiota of patients with acne and $\mathrm{HCs}$}

A total of 7303 OTUs were detected in the samples from the patients with acne and HCs. By comparing the OTUs between samples from the 2 groups, we found 2458 shared OTUs, 3833 OTUs which were unique to acne samples, and 1011 OTUs which were unique to HC samples (Figure 4A). OTU535601 (Lachnospiraceae), OTU4460604 (Clostridiales), OTU3217705 (Moraxellaceae), OTU1066814 (Prevotella), and OTU455671 (Lactococcus 
A

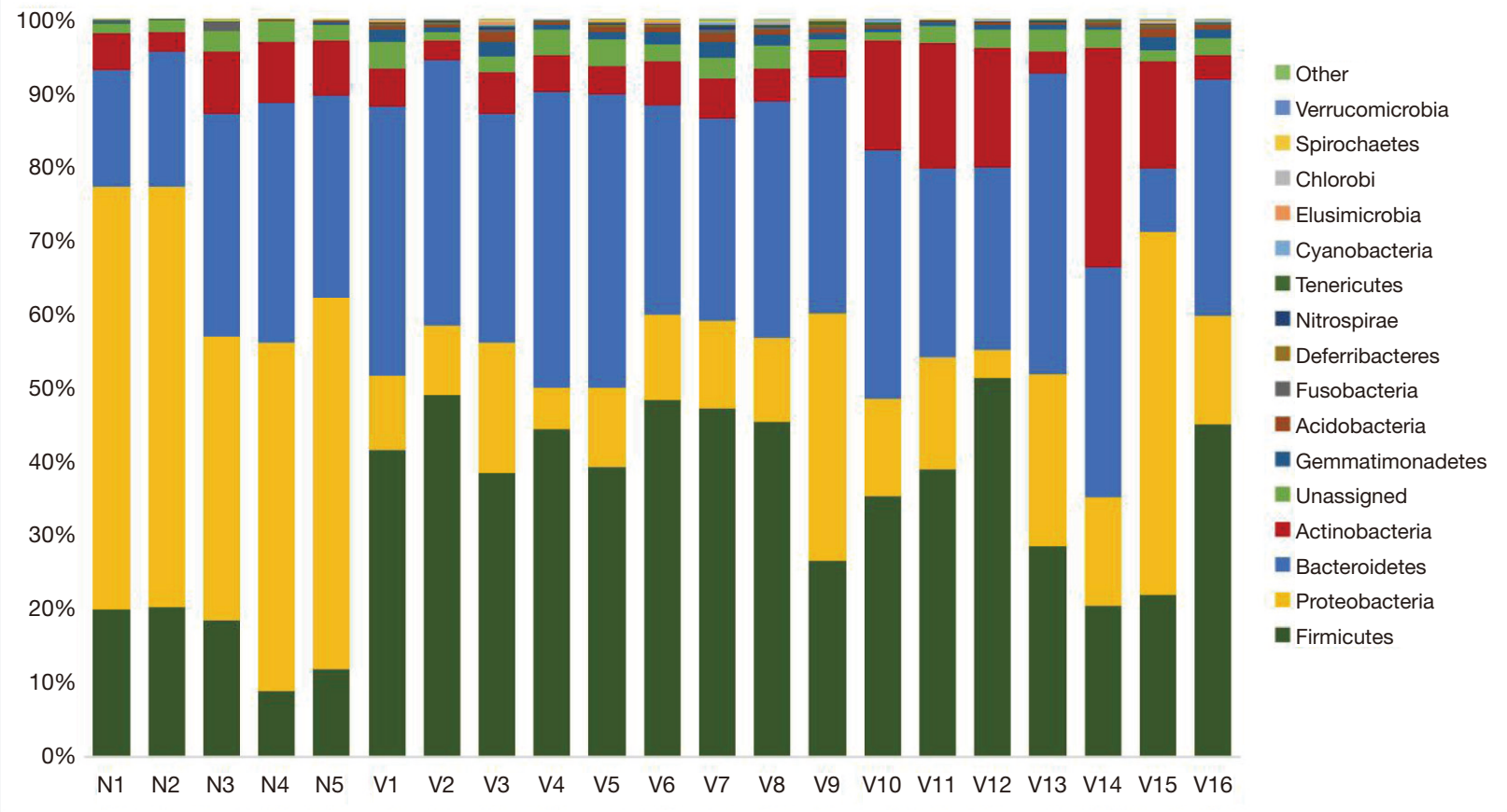

B

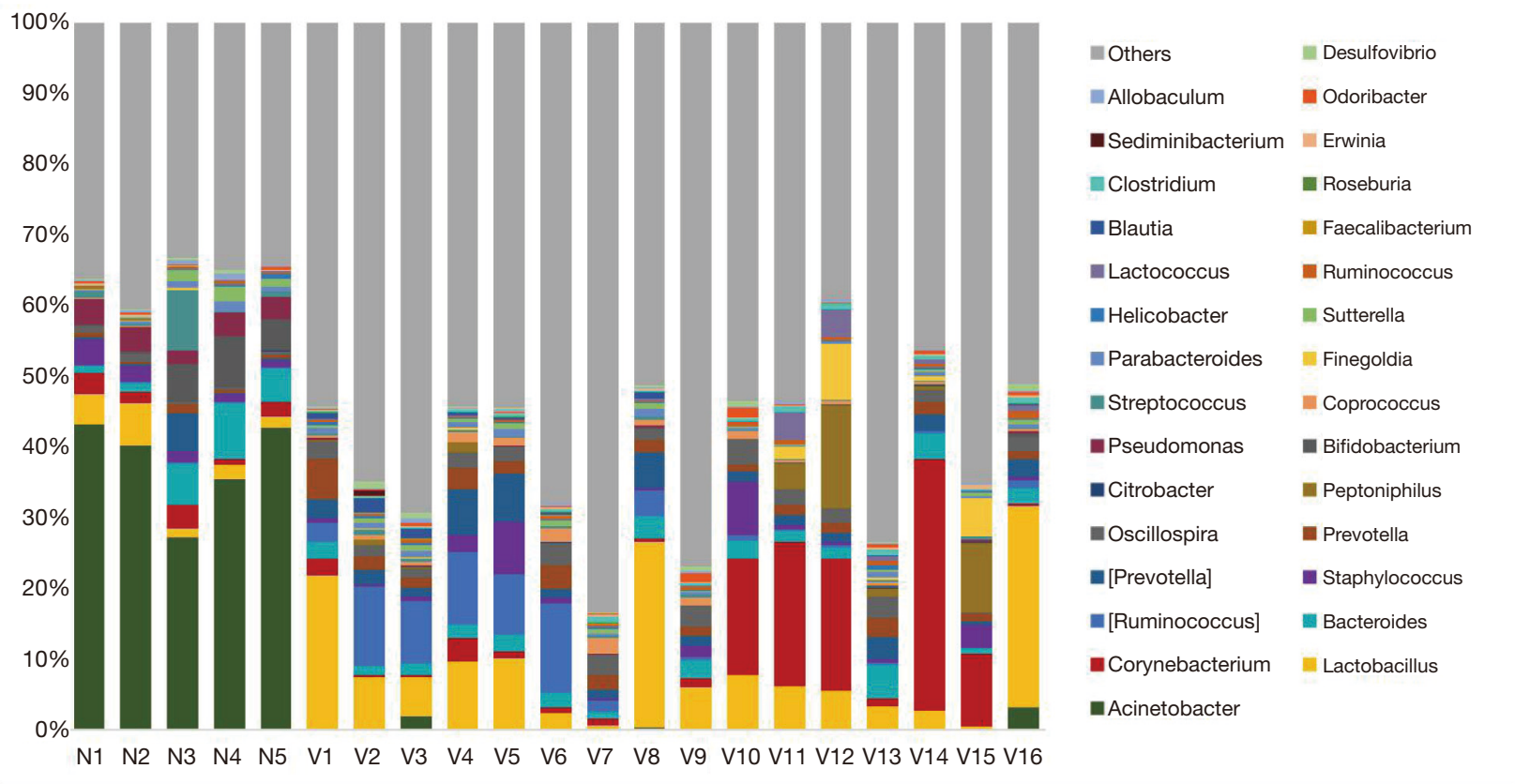

Figure 3 Bacterial community composition of samples. (A) Bacterial community composition at the phylum level. (B) Bacterial community composition at the genus level. 
A

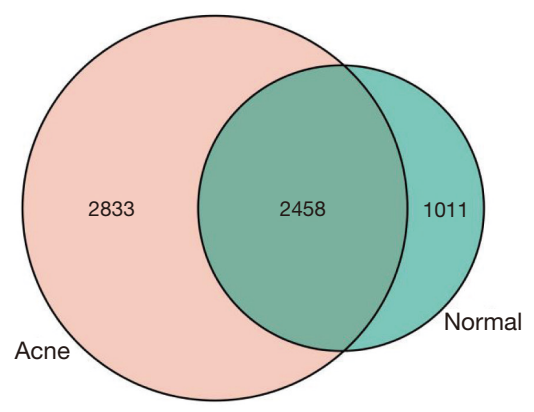

C

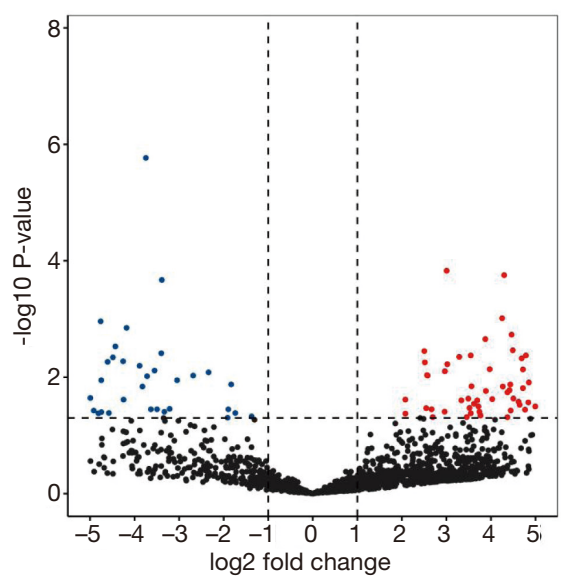

B

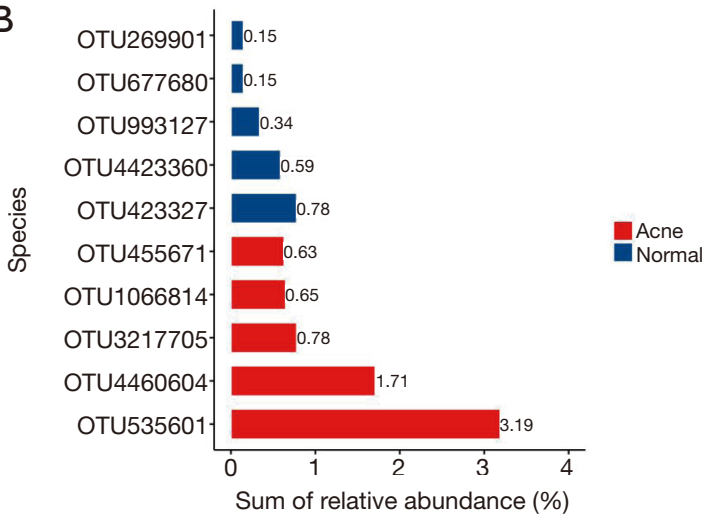

$\mathrm{D}$

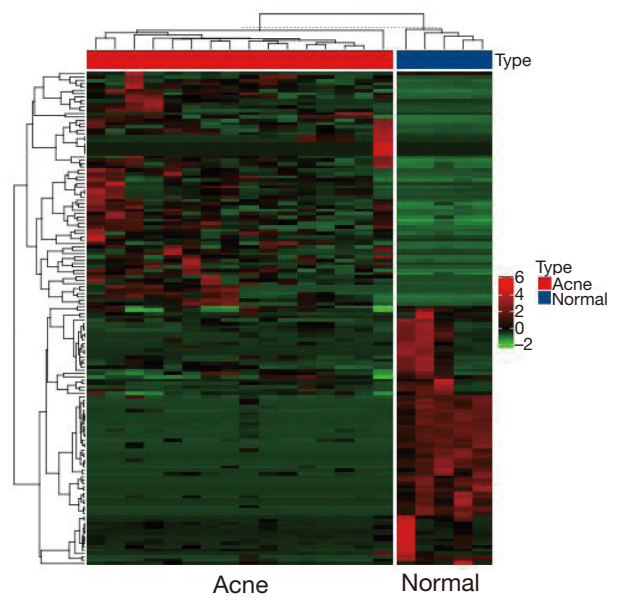

Figure 4 OUT-level analysis of the skin microbiota of patients with acne and HCs. (A) Venn analysis of bacterial OTU composition between acne and HC samples. (B) Top 5 most unique OTUs in the skin microbiota of patients with acne and HCs. (C) Volcano plot of differentially expressed OTUs. Red indicates upregulated OTUs in the acne group; blue indicates downregulated OTUs in the acne group; and black indicates OTUs with no differences between the acne and HC groups. (D) Heatmap showing the abundance of significantly differential OTUs between the acne group and HC group. OTU, operational taxonomic unit; HC, healthy control.

garvieae) were the top 5 most abundant species in the acne group but were not found in the HC samples. OTU423327 (Achromobacter), OTU4423360 (Stenotrophomonas), OTU993127 (Porphyromonas), OTU677680 (Prevotella), and OTU269901 (Pseudomonas) were the top 5 most abundant species in the HC group but were not found in samples from the acne group (Figure 4B). To distinguish the specific characteristic bacteria in the 2 groups, highquality sequence data were analyzed with the Statistical Analyses of Metagenomic Profiles (STAMP) software (Python 2.6.4). Compared with HC samples, samples from patients with acne exhibited 148 markedly altered OTUs $(\mathrm{P}<0.05)$, of which 69 were increased and 79 were decreased (Figure $4 C$ ). The samples from the acne and HC groups could be accurately distinguished based on the characteristics of the differentially expressed OTUs (Figure 4D).

\section{Discussion}

The treatments of acne include topical drugs, oral drugs and physical therapy. Different treatments are selected according to the different grades of acne and the manifestations of different skin lesions. At present, topical antibacterial preparations and oral antibacterial drugs, such as benzoyl peroxide, fusidic acid, and tetracyclines, still have a prominent position in the treatment of acne. In physical therapy, the red/blue light and photodynamic 
therapy can treat and prevent acne by killing $P$. acnes. The skin microbiome is critical to skin health, and any change in the abundance or activities of skin microbiota in response to environmental variations in the host can potentially result in diseases $(2,13)$. In recent years, high-throughput sequencing (next-generation sequencing) technologies, such as denaturing gradient gel electrophoresis, temperature gradient gel electrophoresis, and $16 \mathrm{~S}$ rRNA have been widely used to analyze the diversity of microorganisms from different sources. The use of these technologies has helped to fill the research gap of microorganisms that cannot be cultured in traditional laboratories, and solve the problems posed by low accuracy, operative complexity, and other issues $(14,15)$. Next-generation sequencing has expanded the use of microbial resources, and also constitutes an effective tool for studying interactions between microorganisms.

In the present study, the skin microbiota composition of patients with acne and HCs were assessed by $16 \mathrm{~S}$ rRNA gene sequencing on the Illumina platform, with primers directed against the variable regions $\mathrm{V} 3-\mathrm{V} 4$. The $16 \mathrm{~S}$ rRNA gene has widely served as a mainstay of sequence-based bacterial analysis for decades (16). Shannon index analyses indicated that the sequencing depth covered rare new phylotypes and most of the microbial diversity. Moreover, the Shannon diversity index suggested that microbial diversity was notably increased in the acne group compared to the HC group. Subsequently, to gain a comprehensive insight into the microbiota composition of patients with acne, bacterial taxa differences between the acne and HC groups were investigated through PCA analysis. The results suggested that the microbial community constitutions of patients with acne and HCs were remarkably different. Interestingly, sampling sites, sex, and acne severity had no significant influence on the composition of skin microbiota in our study.

To further investigate the differences in bacterial composition between the 2 groups, bacterial taxa of samples at the phylum and genus levels were plotted. Our findings revealed that in patients with acne, the abundance of Firmicutes was increased and that of Proteobacteria was decreased; further, at the genus level, Acinetobacter showed a sharp reduction, while many other species showed considerable turbulence. Next, we focused on skin microbiota at the OTU level, and found 2458 OTUs that were shared between the acne and HC samples, and 3833 and 1011 OTUs that were unique to acne samples and HC samples, respectively. OTU535601 (Lachnospiraceae), OTU4460604 (Clostridiales),
OTU3217705 (Moraxellaceae), OTU1066814 (Prevotella), and OTU455671 (Lactococcus garvieae) were the top 5 most abundant species in patients with acne but were not found in the HC samples. Enrichment of Lachnospiraceae and Clostridiales has been reported under ultraviolet-B radiation exposure (17), while Moraxellaceae have been found to contribute to mucosal inflammation (18). In a previous study, significant enrichment of Prevotella was observed in the skin microbiota in patients with atopic dermatitis (19). Zhang et al. reported Lactococcus garvieae to be a potential pathogenic cause of skin ulceration syndrome outbreak (20). Based on this evidence, the top 5 most abundant species found on the skin of the patients in our study may be key bacterial factors for the development of acne vulgaris. Additionally, OTU423327 (Achromobacter), OTU4423360 (Stenotrophomonas), OTU993127 (Porphyromonas), OTU677680 (Prevotella), and OTU269901 (Pseudomonas) were the top 5 most abundant species in the HC group but were not found in samples from the acne group. Further analysis of highquality sequence data demonstrated that the samples from the acne group had 148 markedly altered OTUs $(\mathrm{P}<0.05)$, of which 69 were increased and 79 were decreased, compared with the HC group samples. Taken together, our findings show that the diversity and abundance of microorganisms, and the characteristics of the main pathogenic bacteria in patients with acne differ from those in healthy individuals. Due to time limitations, the number of samples can be increased in future studies.

\section{Conclusions}

This study has demonstrated that the composition of the microbial community on the skin of patients with acne differs significantly from that in HCs. Patients with acne vulgaris exhibit increased diversity of skin microbiota and an increased abundance of Lactococcus garvieae compared to healthy individuals.

\section{Acknowledgments}

Funding: This work was financially supported by the Nantong Science Technology Bureau (grant No. MSZ18092) and Nantong Youth Medical Key Talent Project.

\section{Footnote}

Reporting Checklist: The authors have completed the 
STROBE reporting checklist. Available at https://dx.doi. org/10.21037/apm-21-1482

Data Sharing Statement: Available at https://dx.doi. org/10.21037/apm-21-1482

Conflicts of Interest: All authors have completed the ICMJE uniform disclosure form (available at https://dx.doi. org/10.21037/apm-21-1482). The authors have no conflicts of interest to declare.

Ethical Statement: The authors are accountable for all aspects of the work in ensuring that questions related to the accuracy or integrity of any part of the work are appropriately investigated and resolved. All procedures performed in this study involving human participants were in accordance with the Declaration of Helsinki (as revised in 2013). The study was approved by the ethics committee of the Second Affiliated Hospital of Nantong University (No: 2019YKS059) and informed consent was taken from all the patients.

Open Access Statement: This is an Open Access article distributed in accordance with the Creative Commons Attribution-NonCommercial-NoDerivs 4.0 International License (CC BY-NC-ND 4.0), which permits the noncommercial replication and distribution of the article with the strict proviso that no changes or edits are made and the original work is properly cited (including links to both the formal publication through the relevant DOI and the license). See: https://creativecommons.org/licenses/by-nc-nd/4.0/.

\section{References}

1. Barnard E, Li H. Shaping of cutaneous function by encounters with commensals. J Physiol 2017;595:437-50.

2. Byrd AL, Belkaid Y, Segre JA. The human skin microbiome. Nat Rev Microbiol 2018;16:143-55.

3. Oh J, Byrd AL, Deming C, et al. Biogeography and individuality shape function in the human skin metagenome. Nature 2014;514:59-64.

4. Chen Z, Qi J, Wei Q, et al. Variations in gut microbial profiles in ankylosing spondylitis: disease phenotyperelated dysbiosis. Ann Transl Med 2019;7:571.

5. Williams HC, Dellavalle RP, Garner S. Acne vulgaris. Lancet 2012;379:361-72.

6. Xu H, Li H. Acne, the Skin Microbiome, and Antibiotic
Treatment. Am J Clin Dermatol 2019;20:335-44.

7. Li CX, You ZX, Lin YX, et al. Skin microbiome differences relate to the grade of acne vulgaris. J Dermatol 2019;46:787-90.

8. Dreno B, Martin R, Moyal D, et al. Skin microbiome and acne vulgaris: Staphylococcus, a new actor in acne. Exp Dermatol 2017;26:798-803.

9. Hayashi N, Akamatsu H, Kawashima M, et al. Establishment of grading criteria for acne severity. J Dermatol 2008;35:255-60.

10. Nossa CW, Oberdorf WE, Yang L, et al. Design of 16S rRNA gene primers for 454 pyrosequencing of the human foregut microbiome. World J Gastroenterol 2010;16:4135-44.

11. Caporaso JG, Kuczynski J, Stombaugh J, et al. QIIME allows analysis of high-throughput community sequencing data. Nat Methods 2010;7:335-6.

12. Cani PD, Possemiers S, Van de Wiele T, et al. Changes in gut microbiota control inflammation in obese mice through a mechanism involving GLP-2-driven improvement of gut permeability. Gut 2009;58:1091-103.

13. Dréno B, Araviiskaia E, Berardesca E, et al. Microbiome in healthy skin, update for dermatologists. J Eur Acad Dermatol Venereol 2016;30:2038-47.

14. Nkongolo KK, Narendrula-Kotha R. Advances in monitoring soil microbial community dynamic and function. J Appl Genet 2020;61:249-63.

15. Trabal N, Mazón-Suástegui JM, Vázquez-Juárez R, et al. Molecular analysis of bacterial microbiota associated with oysters (Crassostrea gigas and Crassostrea corteziensis) in different growth phases at two cultivation sites. Microb Ecol 2012;64:555-69.

16. Johnson JS, Spakowicz DJ, Hong BY, et al. Evaluation of 16S rRNA gene sequencing for species and strain-level microbiome analysis. Nat Commun 2019;10:5029.

17. Bosman ES, Albert AY, Lui H, et al. Skin Exposure to Narrow Band Ultraviolet (UVB) Light Modulates the Human Intestinal Microbiome. Front Microbiol 2019;10:2410.

18. Hillel AT, Tang SS, Carlos C, et al. Laryngotracheal Microbiota in Adult Laryngotracheal Stenosis. mSphere 2019;4:00211-19.

19. Liu Y, Wang S, Dai W, et al. Distinct Skin Microbiota Imbalance and Responses to Clinical Treatment in Children With Atopic Dermatitis. Front Cell Infect Microbiol 2020;10:336.

20. Zhang Z, Xing R, Lv Z, et al. Analysis of gut microbiota 
revealed Lactococcus garviaeae could be an indicative of skin ulceration syndrome in farmed sea cucumber Apostichopus japonicus. Fish Shellfish Immunol 2018;80:148-54.

Cite this article as: Shi J, Cheng JW, Zhang Q, Hua ZX, Miao $X$. Comparison of the skin microbiota of patients with acne vulgaris and healthy controls. Ann Palliat Med 2021;10(7):79337941. doi: 10.21037/apm-21-1482
(English Language Editor: J. Reynolds) 
Supplementary

Table S1 Sample collection sites information

\begin{tabular}{ll}
\hline ID & Sampling site \\
\hline N1 & cheek \\
N2 & cheek \\
N3 & cheek \\
N4 & forehead \\
N5 & forehead \\
V1 & cheek \\
V2 & forehead \\
V3 & neck \\
V4 & forehead \\
V5 & cheek \\
V6 & cheek \\
V7 & forehead \\
V8 & forehead \\
V9 & brow \\
V10 & forehead \\
V11 & forehead \\
V12 & cheek \\
V13 & neck \\
V14 & forehead \\
V15 & chin \\
V16 & chest \\
\hline
\end{tabular}

Table S2 ANOSIM results

\begin{tabular}{lcc}
\hline ANOSIM results & $\mathrm{R}$ & $\mathrm{P}$ \\
\hline Group (normal/acne) & 0.812 & 0.001 \\
Sex (male/female) & 0.063 & 0.214 \\
Body parts & -0.068 & 0.692 \\
Disease severity & -0.060 & 0.686 \\
\hline
\end{tabular}

